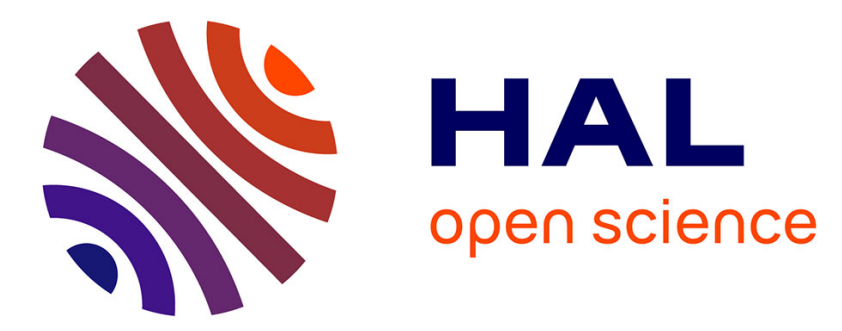

\title{
Personality Traits Do Not Predict How We Look at Faces
}

Charlotte Harrison, Nicola Binetti, Antoine Coutrot, Alan Johnston, Isabelle Mareschal

\section{- To cite this version:}

Charlotte Harrison, Nicola Binetti, Antoine Coutrot, Alan Johnston, Isabelle Mareschal. Personality Traits Do Not Predict How We Look at Faces. Perception, 2018, 47 (9), pp.976-984. 10.1177/0301006618788754 . hal-02348553

\section{HAL Id: hal-02348553 \\ https://hal.science/hal-02348553}

Submitted on 7 Nov 2019

HAL is a multi-disciplinary open access archive for the deposit and dissemination of scientific research documents, whether they are published or not. The documents may come from teaching and research institutions in France or abroad, or from public or private research centers.
L'archive ouverte pluridisciplinaire HAL, est destinée au dépôt et à la diffusion de documents scientifiques de niveau recherche, publiés ou non, émanant des établissements d'enseignement et de recherche français ou étrangers, des laboratoires publics ou privés. 
Personality traits do not predict how we look at faces

Charlotte Harrison ${ }^{1}$, Nicola Binetti ${ }^{1}$, Antoine Coutrot ${ }^{2}$, Alan Johnston ${ }^{1,2,3}$, Isabelle Mareschal $^{4}$

${ }^{1}$ Department of Experimental Psychology, University College London, UK

${ }^{2}$ CoMPLEX, University College London, UK

${ }^{3}$ School of Psychology, University of Nottingham, UK

${ }^{4}$ School of Biological and Chemical Sciences, Psychology, Queen Mary University of

London, UK 


\begin{abstract}
While personality has typically been considered to influence gaze behaviour, literature relating to the topic is mixed. Previously, we (Binetti et al. 2016) found no evidence of self-reported personality traits on preferred gaze duration between a participant and a person looking at them via a video. In the current study, 77 out of the original 498 participants answered an in-depth follow-up survey containing a more comprehensive assessment of personality traits (Big Five Inventory) than was initially used, to check whether earlier findings were caused by the personality measure being too coarse. In addition to preferred mutual gaze duration, we also examined two other factors linked to personality traits: number of blinks and total fixation duration in the eye region of observed faces. Using a multiple regression analysis we found that overall, personality traits do not predict how we look at faces. We suggest that effects previously reported in the literature may stem from contextual differences and/or modulation of arousal.
\end{abstract}


Gaze is a vital component of human interaction. In both infancy and adulthood, people prefer to look at faces compared to any other feature in visual scenes (Birmingham, Bischof \& Kingstone, 2009; Coutrot \& Guyader, 2014). In many contexts, the eyes are amongst the most salient facial features (Yarbus, 1976; Maurer \& Salapatek, 1976). The eyes are important in facilitating successful social interaction and when the eyes are occluded, face recognition (McKelvie, 1976) and detection (Lewis \& Edmonds, 2003) performance drops. Gaze also influences the perception of gender (Schyns, Bonnar \& Gosselin, 2002) and emotion (Calder, Young, Keane \& Dean, 2000). Findings across experiments are constant and seemingly task-independent, suggesting that information from the eye region is critical for multiple aspects of facial processing, including recognition of identity, age, gender and expression (Itier \& Batty, 2009).

There are also known asymmetries in the processing of direct, compared to averted, gaze. The "eye contact effect" refers to any cognitive, perceptual or behavioural change that occurs because of the processing of direct gaze (Senju \& Johnson, 2009). When compared to averted, direct gaze evokes stronger galvanic skin responses (GSR - a proxy of arousal) (Nichols \& Champness, 1971), facilitates face recognition (Hood, Macrae, Cole-Davies, \& Dias, 2003; Vuilleumier, George, Lister, Armony, \& Driver, 2005), and gender categorisation (Macrae, Hood, Milne, Rowe, \& Mason, 2002). Direct gaze is also known to elicit avoidance responses. Ellsworth, Carlsmith and Henson (1972) found that staring confederates on motorcycles caused motorists to depart more rapidly from traffic lights, with comparable results obtained with pedestrians. There have also been multiple findings that more submissive animals will avert gaze, reducing the likelihood of attack by another animal (Cook \& Argyle, 1976). These findings support the idea that prolonged direct gaze is an aversive and threatening signal in many animals (Emery, 2000). 
Gaze preference in humans is often linked to anxiety of the gazer, although the relationship between trait characteristics such as anxiety and neuroticism, and gaze, are mixed. Perlmann et al. (2009) found that participants who scored highly on neuroticism fixated longer in the eye-region of fearful faces compared to those with low levels of neuroticism. Similarly, an earlier study found that in an interview with 'awkward silences', interviewees with low self-concepts and high scores on neuroticism and trait anxiety reacted with increased gaze toward the interviewer (Wiens, Harper, \& Matarazzo, 1980). Conversely, Uusberg, Allik and Hietanen (2015) found that higher neuroticism scores were behaviourally correlated with direct gaze avoidance and subjective averted gaze preference, as well as avoidance-related, relative right-sided functional brain asymmetry. Other studies (e.g. Hobson et al., 1973) found no significant differences.

A study by Roelofts, Putman, Schouten, Lange, Volman \& Rinck (2010) reported that gaze direction mediates processing of emotional faces in anxious individuals. When asked to 'approach' (push a joystick towards) or 'avoid' (push a joystick away) faces with varying expressions, participants with high social anxiety were faster in conditions avoiding angry faces. This avoidance tendency was only present when the perceived anger was directed towards the participant (direct gaze) rather than being directed elsewhere (averted gaze). However, the differences were only found in the 'withdrawal' aspect factor of neuroticism, which may explain previous mixed findings. Reciprocally gaze direction in emotional faces is also influenced by social anxiety, with anxious participants more likely to report gaze directed at them over a wider range of gaze deviations than controls (Jun et al. 2013).

Compared to other personality traits, individual differences in extraversion are typically expected to be most clearly evident in a broad range of self-reported, non-verbal behaviour; in comparison to introverts, extraverts report using more eye contact, vocal 
variety, gesticulation, and facial expression (France, Heisel \& Beatty, 2004). Exline (1963) reported that women with high scores on a measure of "need for affiliation" gazed more at other women in cooperative rather than competitive interactions whereas women with a low "need for affiliation" gazed more at other women in a competitive interaction. Need for affiliation scores were not related to gazing behaviours of men. In face-to-face situations, Mobbs (1968) found that extraverts invest more time looking at a person's eyes, measured by both total and average gaze duration compared to introverts, while Argyle and Ingham (1972) found evidence for correlated extraversion scores and mutual gaze in English, but not Swedish, subjects. Extraverts have been shown to look more frequently at faces than introverts, but there were no differences between the two groups in the proportions of time spent in looking at the others' face and eye contact, or in the mean length of looks. Another study of extraversion showed that extraverts were more likely than introverts to maintain longer gazes while listening during social interaction (Iizuka, 1992). However, more recent research shows that correlations between extraversion and nonverbal behaviour in the literature range between an $r$ of .73 to -.36 (France et al., 2004). When sample size and variance is accounted for, this correlation drops to .13. In addition, some of the earlier results may be influenced by the fact that extraverts are more likely to speak up in-group conversations and eye contact normally accompanies speech (Rutter, Morley \& Graham, 1972).

In additional to the direction of gaze, there are also reported links between personality and blinking habits, with increased blink rate associated with higher neuroticism scores (Barbato, Della Monica, Constanzo \& De Padova, 2012). It has been suggested that blink rate may be modulated by muscle tension, and this may be the mediating link between blink rate and neuroticism (Martin, 1958). Other studies dispute this link, however, with one study only finding a link to the personality trait of psychoticism, and not to any other traits (Colzato et 
al., 2009). The above studies, while mixed, seem to suggest links between personality and gazing behaviour (either in direction of gaze, blink rates or eye contact), which warrants further investigation (Kleinke, 1986).

In our initial exploratory experiment conducted at the London Science Museum we measured the period of mutual gaze that individuals are comfortable with, and examined how that might differ across age, culture and personality. Participants completed a psychological task where they had to judge whether duration of gaze by an actor seen in a video clip felt too long or two short to be comfortable. A staircase procedure was used, and eye tracking-data were collected to also examine differences in scan-path behaviour. Afterwards participants answered a brief questionnaire with demographic information and a short form version of the Big Five Inventory (Rammstedt, \& John, 2007). Our initial study found that preferred gaze duration was not dependent on gender, personality or attractiveness. However, pupil dilation correlated with the amount of eye contact individuals found comfortable; those who preferred longer durations of eye contact displayed faster increases in pupil size when viewing the face than those who preferred shorter durations. This suggested that physiological indices might play an involuntary role in modulating gaze behaviour. The overall preferred gaze duration was found to be 3.3 seconds $( \pm .71)$.

While we previously failed to find a difference in preferred mutual gaze duration and personality, we did not look at other key indicators, such as fixation locations or durations (Binetti, et al. 2016). Further, in our earlier study only a 10-item, short-form version of the Big Five Inventory (Rammstedt \& John, 2007) was used to measure personality scores. This questionnaire might have been too coarse to pick up on individual differences, as traits were only being measured by two items each. 
Here we followed-up on our exploratory approach and sought to investigate whether the use of more exhaustive personality inventory (the full Big Five Inventory) would reveal differences, both in terms of participants' preferred gaze duration with an actor on screen, and their own gaze behaviour. Based on mixed evidence in the literature as well as our earlier result, we hypothesise that personality traits are not strongly related to preferred gaze duration. However, we expect that blink count, average saccade amplitude and mean fixation duration may be linked to personality traits of extraversion and neuroticism.

\section{Method}

\section{Participants}

Our original participant sample consisted of 498 visitors (224 male, 56 nationalities) to the London Science Museum, $(\mathrm{M}=29.9, \mathrm{SD}=12.3$, range $=11-79$ years $)$. Written consent was obtained from participants prior to the experiment. 105 participants responded to the follow-up questionnaire that was sent via email; 15 were excluded from the analysis as their behavioural data for preferred gaze duration could not be reliably fitted to a psychometric function, and a further 13 were removed for having no available or poor eye-tracking data. This left a sample of 77 participants, 37 of which were male (age $\mathrm{M}=33.1, \mathrm{SD}=13.6$, range = 16-72 years). The experiment was approved by the UCL Research Ethics Committee and by the London Science Museum.

\section{Experimental setup}

Behavioural task. Full details are available at Binetti et al. (2016). In brief, participants were shown a series of 40 video clips of an actor who could be male or female 
(chosen randomly from a set of 8 , between the ages of 20-33 years, Caucasian with neutral expressions) gazing directly at them for varying lengths of time. Participants indicated via keyboard response whether the actor's gaze felt too long or too short to feel 'comfortable' (see Figure 1). Video clips were edited in Premiere Pro (CS6, Adobe; www.adobe.com) so the eye region roughly occupied an equivalent area on the screen and the bridge of the nose (nasion) of all actors was aligned with the screen centre. There were 35 possible clips with a period of direct eye contact ranging from $100 \mathrm{~ms}$ to $10,300 \mathrm{~ms}$ (300ms increments, with $\mathrm{ms}$ control). The direct gaze was preceded and followed by 500ms averted downward gaze. Prior to each trial, the nasion position was cued by a black central fixation cross-presented on a grey background to ensure homogeneity in participants' first fixation. Participants gave their response via a key press response after which, a grey screen with the fixation point appeared for $1 \mathrm{~s}$ prior to the next trial commencing. Clip duration was selected based on randomly perturbed estimates yielded by two interleaved QUEST adaptive staircase routines.

Figure 1. On each trial, an actor directly gazes at the participant for a variable amount of time. Participants indicate at the end of the clip whether the actor's direct gaze was 'too short' or 'too long' to feel comfortable.

\section{Results}

Personality data. The relationship between participants' original BFI-10 scores and full BFI scores were examined. A significant positive correlation was found between all ratings: extraversion $(\mathrm{r}=.655, \mathrm{p}<.001)$, conscientiousness $(\mathrm{r}=.600, \mathrm{p}<.001)$, neuroticism $(\mathrm{r}=.712, \mathrm{p}<.001)$, openness $(\mathrm{r}=.285, \mathrm{p}=.006)$ and agreeableness $(\mathrm{r}=.539, \mathrm{p}<.001)$, indicating that self-reported personality traits remained stable over time. See Figure 2 for probability density estimates of self-reported scores. 
Figure 2. Probability density estimates for the five personality traits measured by the Big Five Inventory in our participants.

Behavioural data. Participants' "too long" responses were plotted as a function of the different gaze durations and a cumulative Gaussian was fit to the data to extract their preferred amount of eye contact, which was taken as the midpoint on the Gaussian function. The original dataset (Binetti et al., 2017) had a mean preferred gaze duration (PGD) of $3,295 \mathrm{~ms} \pm 706 \mathrm{~ms}$, mode $=2,800 \mathrm{~ms}$. The current dataset had a mean PGD of $3,550 \mathrm{~ms} \pm$ 1,312ms, and mode of 3,100ms (Figure 3).

Figure 3. Distribution of preferred Gaze Durations.

We performed a multiple regression on the preferred gaze duration, using the 5 personality traits as predictors. Initial correlational analysis revealed that openness was the factor most likely to affect preferred gaze duration, therefore it was the first factor entered into the multiple regression. This was followed by extraversion, then agreeableness, conscientiousness and neuroticism. We found that openness only significantly (weakly) predicted preferred gaze duration $\mathrm{F}(1,76)=4.022$, $\mathrm{p}<0.49$. Given the exploratory nature of this study and the small magnitude of this effect, it must be considered with care.

Eye data. Eye position signals were parsed into fixations and saccades with a custom algorithm based on Nyström \& Holmqvist (2010; see Coutrot et al., 2016 for details). The range of samples lost, and the saccade velocity before and after loss of signal were used to determine blinks. Only blinks during the trials were counted. As the overall length of the experiment varied due to QUEST procedure, each participant's blink count was normalised by dividing the total number of blinks by the total duration of the trials. The average number 
of blinks for a participant was 15 over the entire experiment (average of 5 minutes). Time spent looking at the eye area was calculated as the proportion of fixations occurring within that region of interest. We performed a multiple regression to examine whether personality traits predicted mean fixation duration but none were significant. None of the personality traits predicted time spent in eye region either, however there was a significant effect of extraversion on blinks $F(1,76)=7.16, \mathrm{p}<0.009$.

\section{Discussion}

The current experiment investigated whether there was a relationship between selfreported personality measures and preferred length of mutual gaze duration. We found a weakly significant effect of openness on preferred gaze duration. Further, participants' personality trait of neuroticism predicted their number of blinks. However, the exploratory nature of this study and the overall weakness of these effects make them vulnerable to type I error and should be interpreted with caution.

Past findings on gaze behaviour and personality have been mixed. It may be that the significant differences that have been found have been influenced by contextual variables. For example, eye contact normally accompanies speech in dyads where extraversion scores correlate positively with amount of conversation (Argyle \& Cook, 1967), and more frequent mutual gaze may be a consequence of this greater likelihood of conversation. Other factors than can affect preferred gaze duration, include conversation subject matter, facial expression and gender of those involved (Argyle \& Cook, 1976; Thayer \& Schiff, 1975). This suggests that gaze may be context-dependent rather than trait-dependent. However, it may be that personality variables have a mediating effect; for example, agreeableness may link to respect for social norms and regulate behaviour to act. It may also be that we use someone else's gaze behaviour as an indicator of their personality, even if it does not truly reflect it (France 
et al., 2004). Further, it may be that our sample size of 77 was underpowered when trying to find significant differences in smaller effects, of below .30.

Our results are partially consistent with Perlman et al. (2009) who reported a significant correlation between amount of time spent in the eye region of fearful faces and higher neuroticism scores. Although we found no change in amount of time in eye region, we did find a significant effect of neuroticism on blinks (which they did not investigate). There are however some significant differences between our methodologies that may also account for this discrepancy with respect to amount of time in eye region. Notably, the only significant finding in Perlman et al.'s (2009) paper came from fearful faces, but not neutral faces (consistent with our result which is based only on neutral faces). Further, they made use of static images compared to our dynamic ones. It has been shown that we process static versus dynamic faces differently (Pitcher et al., 2011).

Finally, our participants were given a task compared to the free viewing condition of the other study, which in itself may alter gaze behaviour (Yarbus, 1976). It has been suggested that the eye contact effects may only occur when the observer knows that he/she is being looked at by another "mind". This kind of mentalizing is probably reduced when facing an image or a non-living stimulus (Myllyneva \& Hietanen, 2015). For instance, Pönkänen, Peltola and Hietanen (2011) showed in an ERP study that the N170 normally associated with face and gaze processing produces different signals for direct and averted gaze, but only in live situations and not virtual. Another recent study indicates that time is perceived as being expanded with direct but not indirect gaze, and only when interacting with real people and not virtual avatars (Jarick, Laidlaw, Nasiopoulos \& Kingstone, 2016), suggesting a modulatory role of arousal. However, avatars, along with photos and video recordings, have been successfully utilised in gaze and face perception literature (Itier \& Batty, 2009). Research 
into the increasing use of technology-mediated conversation (such as video conferencing) and level of emotional engagement suggest that stimuli viewed on a screen can be arousing and socially engaging if it is reciprocal (McClure, 2013). Therefore future research should explore

whether robust gaze preference / personality trait relationships can be observed in a reciprocal gaze paradigm, whether face-to-face or via video conferencing.

The current study found that overall, personality traits do not influence face exploration, with the exception of openness weakly predicting preferred gaze duration. Future experiments should focus on contextual factors that may mediate personality traits and on how stimulusrealism and arousal may influence gaze behaviours of both the observer and observed. While gaze behaviour is a clearly important factor in clinical conditions such as autism spectrum disorders and schizophrenia (Itier \& Batty, 2009), the link between personality and gaze may be more complicated.

\section{References}

Argyle, M., \& Cook, M. (1976). Gaze and mutual gaze. Cambridge: Cambridge University Press.

Argyle, M., \& Ingham, R. (1972). Gaze, mutual gaze, and proximity. Semiotica, 6(1), 3249.

Barbato, G., della Monica, C., Costanzo, A., \& De Padova, V. (2012). Dopamine activation in Neuroticism as measured by spontaneous eye blink rate. Physiology \& Behavior, 105(2), 332-336.

Binetti, N., Harrison, C., Coutrot, A., Johnston, A., \& Mareschal, I. (2016). Pupil dilation as an index of preferred mutual gaze duration. Royal Society Open Science, 3(7), 160086.

Birmingham E., Bischof, W.F. \& Kingstone A. Saliency does not account for fixations to 
eyes within social scenes. Vision Research 2009;49:2992-3000.doi:10.1016/j.visres.2009.09.014.

Calder, A. J., Young, A. W., Keane, J., \& Dean, M. (2000). Configural information in facial expression perception. Journal of Experimental Psychology: Human Perception and Performance, 26(2), 527.

Colzato, L. S., Slagter, H. A., van den Wildenberg, W. P., \& Hommel, B. (2009). Closing one's eyes to reality: Evidence for a dopaminergic basis of psychoticism from spontaneous eye blink rates. Personality and Individual Differences, 46(3), 377-380.

Coutrot A, Guyader N. How saliency, faces, and sound influence gaze in dynamic social scenes. Journal of Vision 2014;14:1-17. doi:10.1167/14.8.5.

Coutrot, A., Binetti, N., Harrison, C., Mareschal, I., \& Johnston, A. (2016). Face exploration dynamics differentiate men and women. Journal of Vision, 16(14), 16-16.

Ellsworth, P. C., Carlsmith, J. M., \& Henson, A. (1972). The stare as a stimulus to flight in human subjects: a series of field experiments. Journal of Personality and Social Psychology, 21(3), 302.

Emery, N. J. (2000). The eyes have it: the neuroethology, function and evolution of social gaze. Neuroscience \& Biobehavioral Reviews, 24(6), 581-604.

Exline, R. V. (1963). Explorations in the process of person perception: visual interaction in relation to competition, sex, and need for affiliation1. Journal of Personality, 31(1), 1-20.

Exline, R. V. (1971). Visual interaction: The glances of power and preference. In Nebraska symposium on motivation. University of Nebraska Press.

Hobson, G. N., Strongman, K. T., Bull, D., \& Craig, G. (1973). Anxiety and gaze aversion in dyadic encounters. British Journal of Social and Clinical Psychology, 12(2), 122129.

lizuka, Y. (1992). Eye contact in dating couples and unacquainted couples. Perceptual and Motor Skills, 75(2), 457-461. 
Itier, R. J., \& Batty, M. (2009). Neural bases of eye and gaze processing: the core of social cognition. Neuroscience \& Biobehavioral Reviews, 33(6), 843-863.

Jarick, M., Laidlaw, K. E., Nasiopoulos, E., \& Kingstone, A. (2016). Eye contact affects attention more than arousal as revealed by prospective time estimation. Attention,Perception, \& Psychophysics, 1-6.

Jun, Y.Y., Mareschal, I., Clifford, C.W.G., \& Dadds, M.R. (2013). Cone of direct gaze is a marker of social anxiety in males. Psychiatry Research, Nov, 210(1), 193-198.

Kleinke, C. L. (1986). Gaze and eye contact: a research review. Psychological Bulletin, 100(1), 78 .

La France, B. H., Heisel, A. D., \& Beatty, M. J. (2004). Is there empirical evidence for a nonverbal profile of extraversion?: a meta-analysis and critique of the literature. Communication Monographs, 71(1), 28-48.

Lewis, M. B., \& Edmonds, A. J. (2003). Face detection: Mapping human performance. Perception, 32(8), 903-920.

Maurer, D., \& Salapatek, P. (1976). Developmental changes in the scanning of faces by young infants. Child Development, 523-527.

McClure, E. (2013, June). Infant emotional engagement in video mediated interactions. In Proceedings of the 12th International Conference on Interaction Design and Children (pp. 655658). ACM.

McKelvie, S. J. (1976). The role of eyes and mouth in the memory of a face. The American Journal of Psychology, 311-323.

Myllyneva, A., \& Hietanen, J. K. (2015). There is more to eye contact than meets the eye. Cognition, 134, 100-109.

Nichols, K. A., \& Champness, B. G. (1971). Eye gaze and the GSR. Journal of Experimental Social Psychology, 7(6), 623-626. 
Nyström, M., \& Holmqvist, K. (2010). An adaptive algorithm for fixation, saccade, and glissade detection in eyetracking data. Behavior Research Methods, 42(1), 188-204.

Perlman, S. B., Morris, J. P., Vander Wyk, B. C., Green, S. R., Doyle, J. L., \& Pelphrey, K. A. (2009). Individual differences in personality predict how people look at faces. PloS One, 4(6), e5952.

Pitcher, D., Dilks, D. D., Saxe, R. R., Triantafyllou, C., \& Kanwisher, N. (2011). Differential selectivity for dynamic versus static information in face-selective cortical regions. Neuroimage, 56(4), 2356-2363.

Pönkänen, L. M., Peltola, M. J., \& Hietanen, J. K. (2011). The observer observed: Frontal EEG asymmetry and autonomic responses differentiate between another person's direct and averted gaze when the face is seen live. International Journal of Psychophysiology, 82(2), 180187.

Rammstedt, B., \& John, O.P. (2007). Measuring personality in one minute or less: A 10item short version of the Big Five Inventory in English and German. Journal of Research in Personality, 41, 203-212.

Roelofs, K., Putman, P., Schouten, S., Lange, W. G., Volman, I., \& Rinck, M. (2010). Gaze direction differentially affects avoidance tendencies to happy and angry faces in socially anxious individuals. Behaviour Research and therapy, 48(4), 290-294.

Rutter, D. R., Morley, I. E., \& Graham, J. C. (1972). Visual interaction in a group of introverts and extraverts. European Journal of Social Psychology,2(4), 371-384.

Schyns, P. G., Bonnar, L., \& Gosselin, F. (2002). Show me the features! Understanding recognition from the use of visual information. Psychological Science, 13(5), 402-409.

Senju, A., \& Johnson, M. H. (2009). The eye contact effect: mechanisms and development. Trends in Cognitive Sciences, 13(3), 127-134.

Uusberg, H., Allik, J., \& Hietanen, J. K. (2015). Eye contact reveals a relationship between Neuroticism and anterior EEG asymmetry. Neuropsychologia, 73, 161-168. 
Wiens, A. N., Harper, R. G., \& Matarazzo, J. D. (1980). Personality correlates of nonverbal interview behavior. Journal of Clinical Psychology, 36(1), 205-215.

Yarbus A. L. (1967). Eye movements and vision. New York: Plenum. 


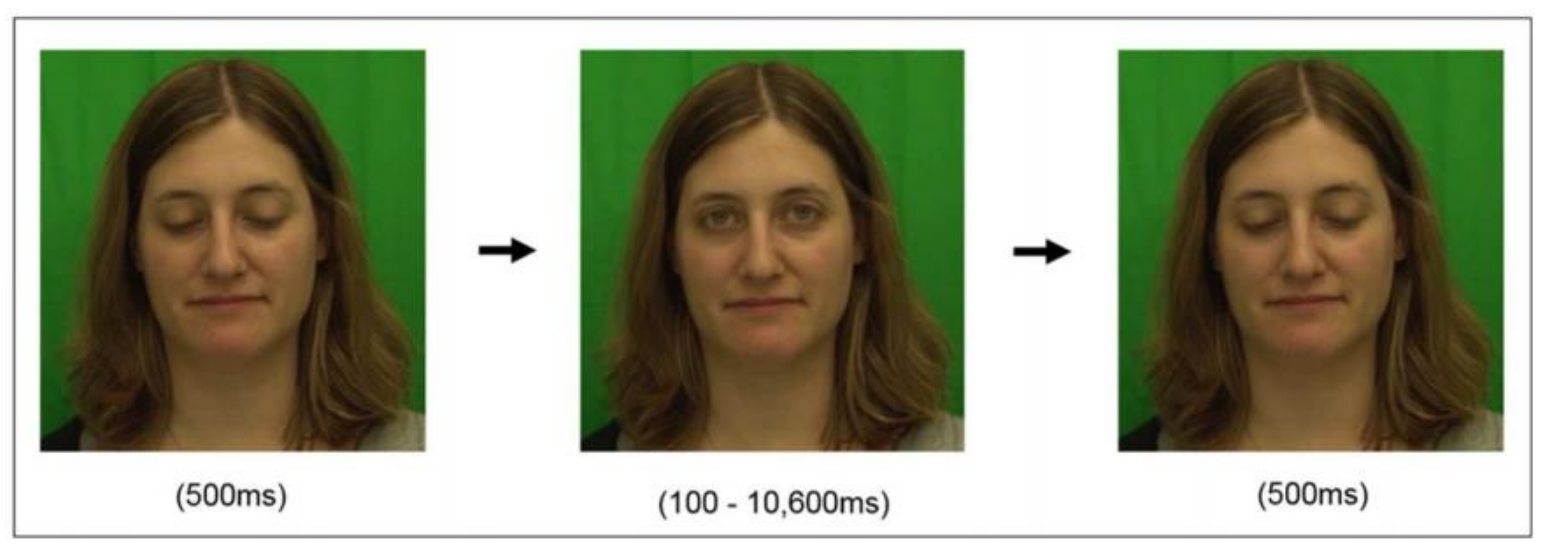

Figure 1

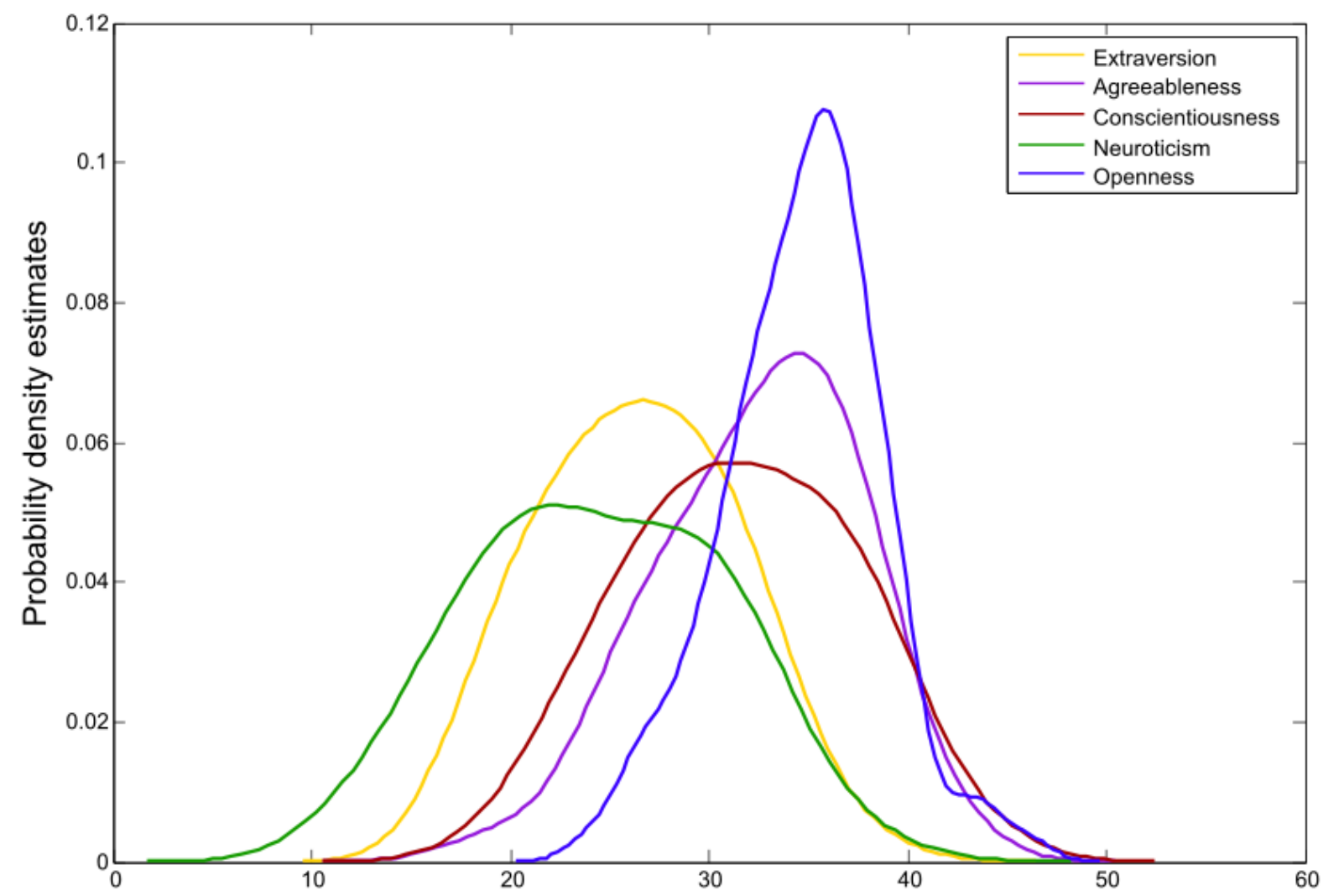

Big Five Inventory Scores

Figure 2 


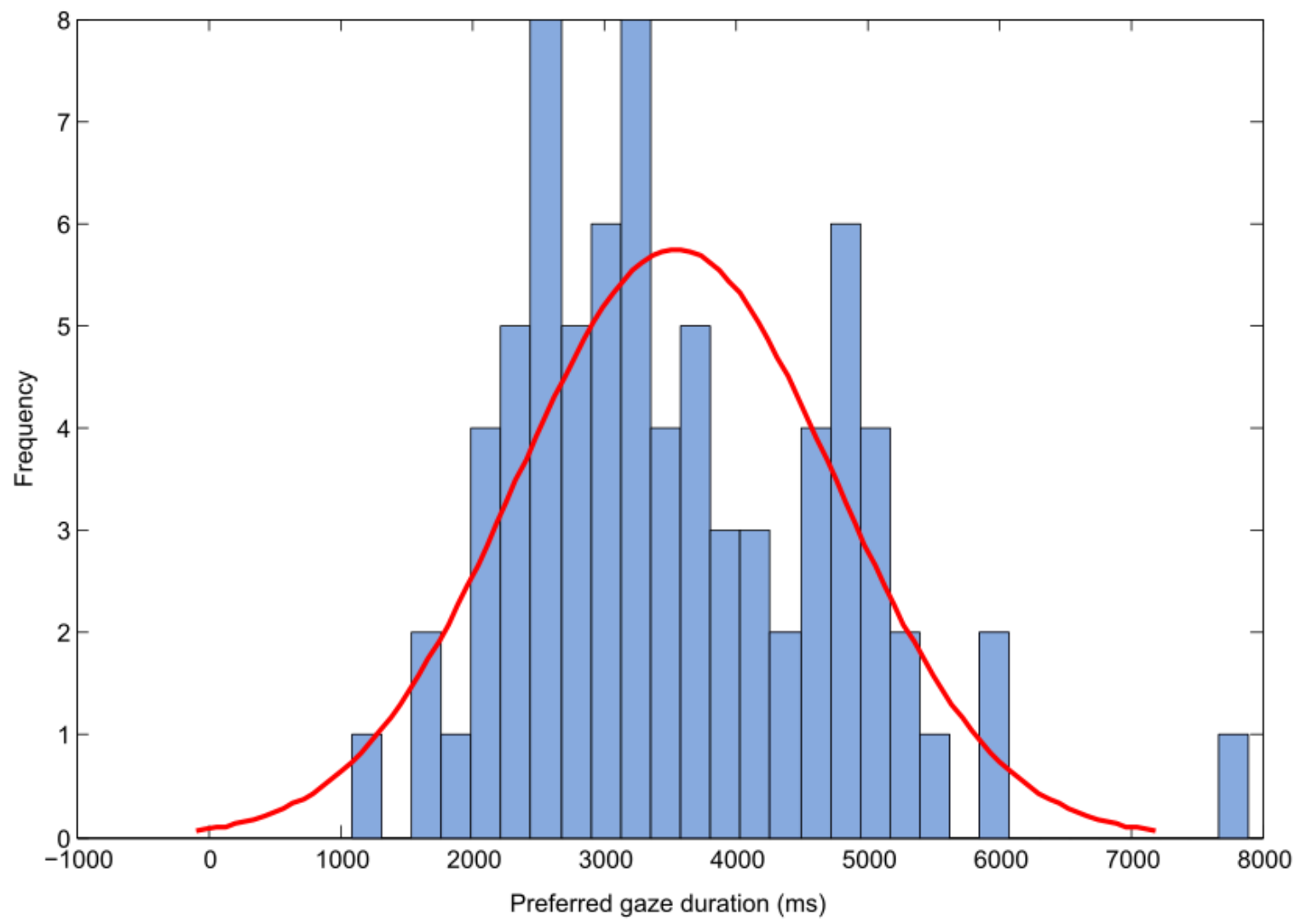

Figure 3 